\title{
Mating changes the female dietary preference in the two-spotted cricket, Gryllus bimaculatus
}

\author{
Yusuke Tsukamoto ${ }^{1,2}$, Hiroshi Kataoka ${ }^{1}$, Hiromichi Nagasawa ${ }^{2}$ and Shinji Nagata ${ }^{1,2 *}$ \\ 1 Department of Integrated Biosciences, Graduate School of Frontier Sciences, The University of Tokyo, Chiba, Japan \\ ${ }^{2}$ Department of Applied Biological Chemistry, Graduate School of Agricultural and Life Sciences, The University of Tokyo, Tokyo, Japan
}

\section{Edited by:}

Ken-ichi Moto, RIKEN, Japan

Reviewed by:

Virginie Tolle, INSERM, France

Joe Hull, USDA Agricultural

Research Service, USA

${ }^{*}$ Correspondence:

Shinji Nagata, Department of Integrated Biosciences, Graduate

School of Frontier Sciences, The

University of Tokyo, Kashiwanoha

5-1-5, Kashiwa city, Chiba 277-8567,

Japan

e-mail: shinjin@k.u-tokyo.ac.jp
Most insect species exhibit characteristic behavioral changes after mating. Typical post-mating behaviors in female insects include noticeable increases in food intake, elevated oviposition rates, lowered receptivity to courting males, and enhanced immune response. Although it has been reported that mated females of several insect species including the fruit fly, Drosophila melanogaster increase the amount of food intake and change their dietary preferences, the limited number of comparative studies prevent the formulation of generalities regarding post-mating behaviors in other insects in particular amongst orthopteran species. Here, we investigated whether females of the two-spotted cricket, Gryllus bimaculatus, alter their feeding behavior after mating. Although significant differences in the amount of food intake after mating were not observed, all experimental data indicated a clear trend among crickets toward the ingestion of larger quantities of food. Geometric framework analyses revealed that the mated female crickets preferred food with higher protein content compared to virgin female crickets. This implies that this species required different nutritional demands after mating. These findings further expand our understanding of the behavioral and biological changes that are triggered in female insects post-mating, and highlight the potential for this species in investigating the molecular-based nutritional dependent activities that are linked to post-mating behaviors.

Keywords: feeding behavior, geometric framework, Gryllus bimaculatus, post-mating behavior

\section{INTRODUCTION}

Most animals, including insects, exhibit a repertoire of behavioral and physiological changes after mating. The characteristic changes in the post-mating behavior in insects have been extensively studied and reported (Wolfner et al., 2005). In general, it has been demonstrated that insects exhibit major behavioral changes immediately after mating, with this phenomenon being more exaggerated in the mated females compared to the mated males. The mated females of the fruit fly, Drosophila melanogaster, exhibit a number of characteristic post-mating behavioral changes such as the stimulation of ovulation and reduction of receptivity for courting males (Wolfner, 1997). In addition, the female immune system is activated in responses to male seminal fluid factors (Lawniczak and Begun, 2004; McGraw et al., 2004; Peng et al., 2005). Similar behavioral changes related to reproduction have also been observed in other insect species, including the common house mosquito, Culex pipiens (Chiba et al., 1992), the Mediterranean fruit fly, Ceratitis capitata (Jang, 2002), in lepidopteran species, such as the cotton bollworm, Helicoverpa armigera (Jin and Gong, 2001), in hemipteran species, such as the western tarnished plant bug, Lygus hesperus (Brent, 2010) and in coleopteran species, such as the ground beetle Leptocarabus procerulus (Takami et al., 2008).

Of the various recorded characteristic post-mating behaviors, motivated feeding behavior has also been reported in several insects (Table 1). For example, after mating, D. melanogaster females increase the amount of food intake (Carvalho et al., 2006). Similarly, motivated feeding behavior has been observed across several insect orders; the two-spotted ladybird, Adalia bipunctata (Perry, 2011) in Coleoptera, the yellow fever mosquito, Aedes aegypti (Judson, 1967) in Diptera and L. hesperus (Cooper and Spurgeon, 2011) in Hemiptera. It has also been demonstrated that the mated females of $D$. melanogaster alter dietary preference (Ribeiro and Dickson, 2010; Vargas et al., 2010). It is likely that the post-mating events including copulation, ejaculation, and oogenesis motivated their feeding and changes the feeding preference in several insect species. Although a number of physiological studies have been performed concerning post-mating feeding behavior, molecular-based studies remain limited.

To date, RNA interference techniques and genomic editing manipulations have proven to be powerful tools for exploring numerous biological phenomena at the molecular level, even in non-model insect species such as the two-spotted cricket, Gryllus bimaculatus (Nakamura et al., 2010; Konuma et al., 2012; Watanabe et al., 2012). Based on previous studies, it has become increasingly evident that the two-spotted cricket represents a good model species for studying the post-mating effects on feeding behavior.

In this study, we investigated whether G. bimaculatus follows the pattern established in other insects regarding the effects of mating on feeding behavior. To address the intriguing issues, we performed several physiological experiments to monitor the feeding behavioral change in adult female G. bimaculatus after mating. 
Table 1 | Post-mating feeding behavior changes in several insect species.

\begin{tabular}{llll}
\hline Order & Species & Post-mating feeding behavioral changes & References \\
\hline Diptera & Drosophila melanogaster & $\begin{array}{l}\text { Increasing the amount of food intake } \\
\text { Increasing the preference of yeast }\end{array}$ & $\begin{array}{l}\text { Carvalho et al., 2006 } \\
\text { Ribeiro and Dickson, 2010; Vargas et al., 2010 } \\
\end{array}$ \\
\cline { 2 - 4 } & Aedes aegypti & Altered the feeding pattern & Judson, 1967 \\
& Glossina austeni & Increasing the amount of blood meal intake & Adlakha and Pillai, 1976 \\
& Culex pipiens & Increasing the amount of blood meal intake & Ejezie and Davey, 1977 \\
Increasing the amount of blood meal intake & Adlakha and Pillai, 1976 \\
Coleoptera & Adalia bipunctata & Increasing the amount of food intake & Perry, 2011 \\
Hemiptera & Lygus hesperus & Spending more time for stylet-probing & Cooper and Spurgeon, 2011 \\
\hline
\end{tabular}

We specifically focused on different feeding parameters, including the amount of food intake and dietary preference.

\section{MATERIALS AND METHODS CHEMICALS AND REAGENTS}

Cellulose powder used in the present study was purchased from Nacalai tesk (Kyoto, Japan). Dextrin hydrate and casein were purchased from Wako Pure Chemical Industries (Osaka, Japan). For cricket food, rabbit ORC4 was purchased from ORIENTAL YEAST Co., Ltd. (Tokyo, Japan) and a commercially available diet of cat food (Friskies dry mix) was purchased from Nestle Purina PetCare Co., Ltd. (Hyogo, Japan).

\section{INSECTS}

Fifth instar larvae of the two-spotted cricket, G. bimaculatus, were purchased from Tsukiyono Farm Co., Ltd. (Gunma, Japan). Crickets were reared in plastic containers $(55 \times 39 \times 31 \mathrm{~cm})$ at $27 \pm 1{ }^{\circ} \mathrm{C}$ with $70 \pm 5 \%$ relative humidity under long-day lighting conditions (16-h light, 8-h dark cycle). Crickets were fed ad libitum on a mixture of rabbit ORC4 and cat food in a ratio of approximately one to one with unlimited access to water. Although most crickets grow and molt simultaneously after arriving at the laboratory, we used crickets with synchronous growth from the last instar to adult emergence. Because the amount of food intake for the first 5 days after adult emergence was remarkably inconstant, we used virgin females 7-10 days after adult emergence. Male crickets used in this study were reared with adult females.

\section{MEASUREMENT OF THE AMOUNT OF FOOD INTAKE}

To measure the amount of food intake, we prepared "commercial" diet tablets made of cellulose powder, rabbit ORC4, and dextrin. Powdered rabbit ORC4 and dextrin were mixed with cellulose powder; rabbit ORC4:dextrin $=2: 1$ (wt/wt). Cellulose powder was added to the mixture at a final concentration of $70 \%$. The mixture was transferred into a plastic template $(\varphi 2.1 \times$ $0.5 \mathrm{~cm}$ ). The mixture was hardened after wetting with water by pushing under hand-pressure, and then frozen at $-80^{\circ} \mathrm{C}$. The frozen tablets were lyophilized to dryness. The resulting tablets were weighed as starting test diets. Before measuring the weight of the remaining tablets after feeding, the water in the tablets was removed by heating at $70^{\circ} \mathrm{C}$ for $3 \mathrm{~h}$. Adult virgin females fed ad libitum were isolated individually in round plastic containers $(\varphi 9.9 \times 9.4 \times 4.5 \mathrm{~cm})$ and acclimated to the synthetic tablet diets for 1 day before experimental treatment. In assays measuring the amount of food intake, adult females kept isolated in the containers were examined for 3 days after acclimation. As the different periods (for 1, 2, and 3 days) of acclimation for dietary tablet do not change the trends of the results, we acclimated for 1 day in the present study. The mated females were prepared by putting adult male crickets into containers with a virgin female. After the end of mating, the males were removed, and then diet tablets were given to the mated females. As the remaining tablets were removed to measure the amount of food intake, new tablets were supplied every day. During this experiment, crickets could freely access water.

\section{FOOD CHOICE TEST}

To test the dietary preference of the crickets, we prepared two different "generic" diet tablets made of cellulose powder, dextrin, and casein. Dextrin and casein were mixed with cellulose powder at different ratios by weight: Carbohydrate-rich dietdextrin: casein (10:1); Protein-rich diet-dextrin: casein (1: 10). Each tablet was adjusted with cellulose powder to a final concentration of $67 \%$. Adult virgin females fed ad libitum were isolated in the round plastic containers and acclimated to the two different diet tablets with free access to water for 1 day before the experimental treatment. After that, adult male crickets were transferred into containers for mating. After the end of mating, the males were removed, and then diet tablets were given to the mated females. As the remaining tablets were removed to measure the amount of food intake, new tablets were supplied every day. Geometric analyses were performed based on the previous description (Raubenheimer and Simpson, 1993; Simpson and Raubenheimer, 1995).

\section{STATICAL ANALYSES}

Comparisons across two groups were statistically analyzed by Student's $t$-test and Dunnett's test. Comparison of food intake in individuals was analyzed by paired $t$-test. $P$-values less than 0.05 were considered to be statistically significant. All experiments were performed at least twice and the authors confirmed that the resulting data were reproducible.

\section{RESULTS}

\section{AMOUNT OF FOOD INTAKE AFTER MATING IN G. BIMACULATUS}

Throughout our observations of the adult female two-spotted cricket, G. bimaculatus under our rearing conditions, we noticed 
that, similar to other insect species, the mated females appeared to increase their food intake (Table 1). To confirm if this species will undergo a change in post-mating feeding behavior; we first measured the amount of food intake by mating in the female adult crickets using "commercial" dietary tablets made of rabbit ORC4, dextrin and cellulose powder. Observation revealed that the amount of food intake in adult females before mating was $345 \pm 113.6 \mathrm{mg} /$ day, while that in adult females after mating was $359 \pm 127.6 \mathrm{mg} /$ day; a statistically significant increase in the amount of food intake was not found (Figure 1A). On the other hand, the mated females ingested more food (359 \pm $127.6 \mathrm{mg} /$ day) compared to control virgin females $(257 \pm 145.7$ $\mathrm{mg} /$ day); but not significantly (Figure 1B).

\section{CHANGES IN DIETARY PREFERENCE AFTER MATING}

Because G. bimaculatus did not show a significant increase of food intake after mating, we sought to explore the details of the slight increase of food intake. We examined whether mated females alter their dietary preference. Mated females were subjected to a choice assay for 3 days using two nutritionally different "generic" diet tablets (carbohydrate-rich and protein-rich diet tablets). Unlike the first experiment (Figures 1A,B), the mated females ate significantly more than virgins when carbohydrate-rich and protein-rich diet tablets were supplied (Figure 2). To evaluate the effects of mating on the dietary preference of adult females, we conducted a geometric framework analysis, which has been frequently used for analyses of dietary preferences (Raubenheimer and Simpson, 1993; Simpson and Raubenheimer, 1995). Mated females were fed ad libitum on both of the tablets for 3 days. The geometric framework analyses by using data from individual crickets indicated that mated females preferred protein-rich diet tablets to carbohydrate-rich tablets compared to the virgin

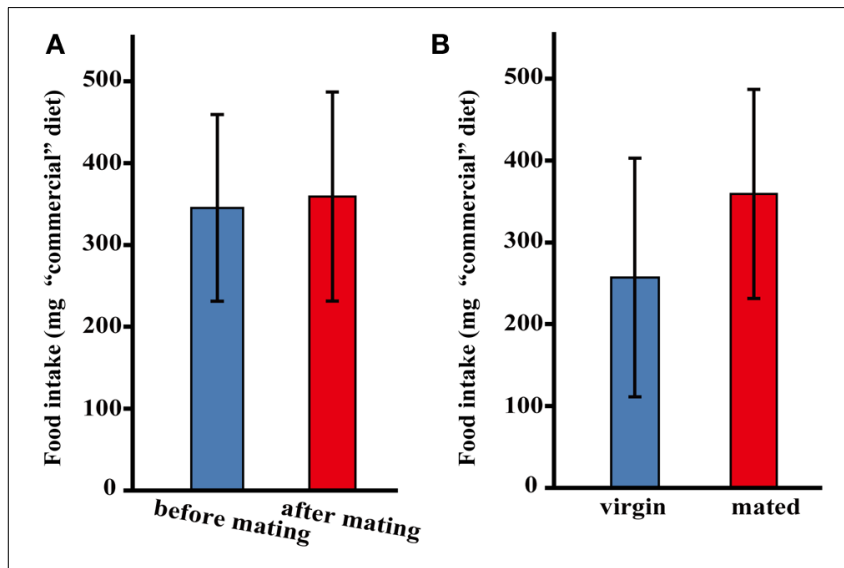

FIGURE 1 | The amount of "commercial" diet intake after mating in G. bimaculatus females. The amount of food intake by $G$. bimaculatus adult females after mating was measured by consumption of "commercial" diet tablets. (A) The amount of food intake by individual females before mating was also measured as a control. $N=8$, Mean $\pm S D$. Data was statistically analyzed using a paired $t$-test. The amount of food intake by females before and after mating was compared. (B) The amount of food intake by the mated females was measured by comparing to the amounts of food intake by the virgin females. Mated females; $N=8$, virgin females; $N=10$, Mean $\pm S D$. Data was statistically analyzed using Dunnett's test. females (Figures 3A,B). However, a number of individuals exhibited distinctive trends in preference from the major population (asterisks in Figure 3A). Even though these individuals ruled out the tendency of preference, we were able to obtain significant differences in different experimental trials. To confirm if mated females exhibit motivated feeding on a protein-rich diet, we next examined the amount of each nutrient consumed. The mated females ate as much dextrin as the virgin females (Figure 4), while mated females ate approximately twice the amount of casein as virgin females (Figure 4).

\section{DISCUSSION}

In the present study, we demonstrated that immediately after mating the adult female did not exhibit a significant increase in the amount of food intake compared to virgin females when tablets made of rabbit ORC4 were used. This contrasts with previous studies on other insect, such as D. melanogaster (Carvalho et al., 2006) and A. bipunctata (Perry, 2011), which reported significant increases in food intake after mating. Although our result was not statistically significant, the similar tendency to increase the amount of food intake was observed in at least five trials of observation. These unexpected results might be caused by using diet tablets made of rabbit ORC4, which is a commercially available artificial diet that contains several nutrients. ORC4 for phytophagous animals might not be as sufficient for omnivorous crickets in satisfying their nutritional demands. In fact, a commercially available cat food provided different data albeit with a similar tendency to motivate feeding after mating. On the other hand, when tablets that contained dextrin and casein as nutrients were used, the amount ingested by mated females increased (Figure 2). These results suggest that the amount of food intake depends on the nutritional contents of the dietary source.

The selection of diet are not only due to the nutritional states but also due to the nutritional contents of food as the previous

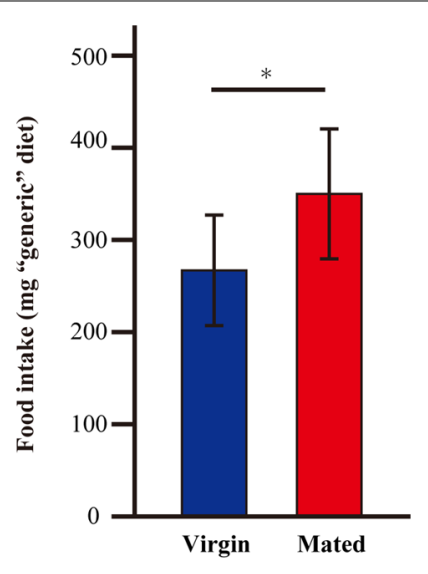

FIGURE 2 | The amount of "generic" diet intake after mating in $\mathbf{G}$. bimaculatus females. The amount of food intake by $G$. bimaculatus adult females after mating was measured by consumption of two different "generic" diet tablets: carbohydrate-rich and protein-rich tablets. The amount of food intake by the mated females was measured and was compared with the amount of food intake by the virgin females. $N=7$, Mean $\pm S D$. Data was statistically analyzed by Student's $t$-test. $P$-values less than 0.05 were considered to be statistically significant. ${ }^{*} P<0.05$. 

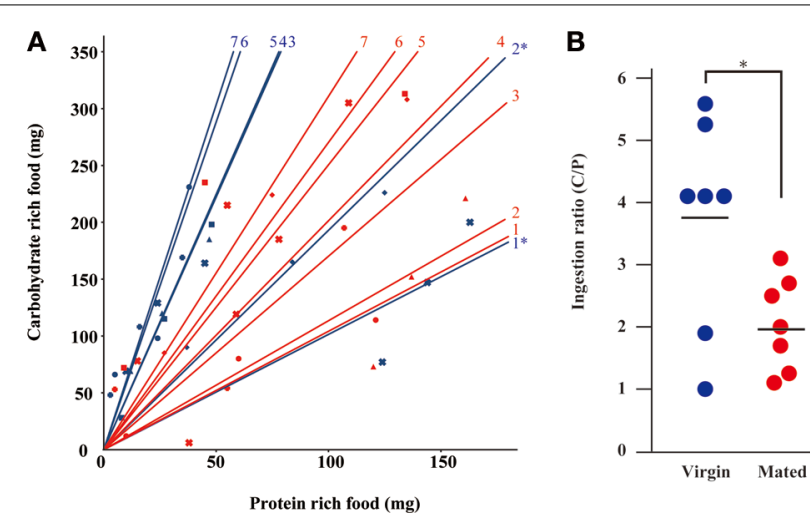

FIGURE 3 | The transition of dietary preference after mating. (A) The geometric framework analysis by mated female crickets. The horizontal axis represents the amount of protein-rich food intake and the vertical axis represents the amount of carbohydrate-rich food intake using "generic" diet tablets. Red and blue spots indicate the measured values from mated and virgin females, respectively. Different symbols indicate different individuals. Red and blue approximate lines indicate mated and virgin females, respectively. Data were plotted as every day. The same symbol indicates the points of the amount of food ingested each day individual. Each data point was plotted as an accumulated value from the first observation day in this figure. The numbers of individuals examined are indicated. An asterisk represents the data from an unusual cricket as described in the text. (B) The ingestion ratio of "generic" casein-rich diet/protein-rich diet (C/P) by mated and virgin females. Dots indicate the data from individual crickets. Bars indicate mean values. Data were statistically analyzed by Student's $t$-test. $P$-values less than 0.05 were considered to be statistically significant. ${ }^{*} P<0.05 . N=7$, Mean $\pm S D$.

report also suggested in other insect species. The elicited preference in protein-rich food by mated females (Figures 3A,B) is reminiscent of that observed in the post-mated $D$. melanogaster females, in which mated females ate more yeast compared to virgin females (Ribeiro and Dickson, 2010; Vargas et al., 2010). If the mated $D$. melanogaster adult females consumed yeast as a protein or nitrogen source, it is consistent with the data obtained by the present observations. This hypothesis is also supported by other recent studies suggesting that protein from the ingested diet by $D$. melanogaster adult females is used as nitrogen source for producing eggs (Tatar, 2011). Therefore, the elicited protein preference after mating might be conserved across insect species, with protein being an essential nutrient for oogenesis and oviposition. In preliminary observations, crickets laid eggs from the first day after mating to early on the second day (data not shown). In contrast, preferential feeding on the high protein diet started to increase late on the second day (Figure 3A). These observations suggest that egg laying is differentially timed to the initiation of protein food preference after mating. Therefore, it is possible that egg laying is strongly linked to the change in dietary preference. $G$. bimaculatus females are able to lay eggs multiple times in their lives. Perhaps, the different amounts of dietary protein ingested by mated females compared to virgin females is directly linked to the demands for generation of the newly developing eggs for subsequent mating and egg laying events.

It is intriguing that imbalanced nutritional states might potentially cause a specific nutritional dependent feeding behavior, as

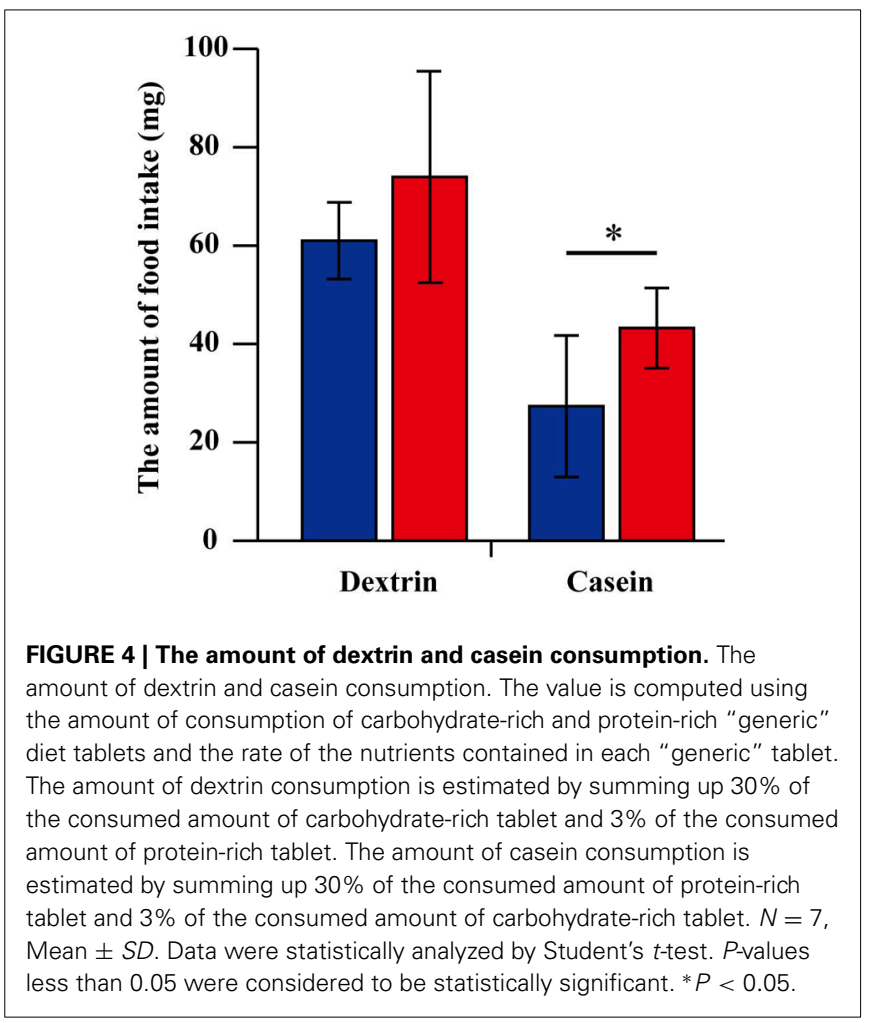

observed in the mated females in this study, and other animal species in previous studies. In the case of post-mating behavior, this change in a possible nutritional state may result from seminal factors penetrating through the ovarian barrier layer (Avila et al., 2011). It has been observed in many insect species that a number of seminal fluid factors influence post-mating events (Gillott, 2003; Avila et al., 2011). For example, the 36 amino acid accessory gland derived $D$. melanogaster sex peptide (SP) is a striking example of a factor influencing mating-activated events (Chen et al., 1988; Kubli and Bopp, 2012). This peptide penetrates through the ovarian layer in the mated females from the ejaculated seminal fluid (Pilpel et al., 2008). Genetic manipulation of SP-related genes has shown that SP causes some changes in the feeding behavior of adult female $D$. melanogaster immediately after mating (Kubli and Bopp, 2012). In addition, SP-like peptides have also been found in some insect species such as Helicoverpa armigera (Nagalakshmi et al., 2007). In H. armigera an SP-like peptide and its receptor mediate pheromone biosynthesis and remating rejection behavior against courting males (Nagalakshmi et al., 2007; Hanin et al., 2011). SP and SP-like peptides are not conserved across insect species. Although SP is not present in some insect species, the SP receptor is highly conserved (Yapici et al., 2008; Kim et al., 2010; Poels et al., 2010). Therefore, further research is required to elucidate whether changes in feeding behavior after mating are directly mediated by SP and SPR mechanisms. The lack of a sequenced $G$. bimaculatus genome makes it difficult to ascertain if SP-like peptides and SPR are present in G. bimaculatus. First, it will be particularly important to confirm and characterize the contribution of seminal factors, including SP and SPR, possibly contributing to changes in the feeding behavior of 
G. bimaculatus, and to determine the mechanisms by which such dramatic changes in the post-mating behavior are affected.

It is interesting that the transition of nutritional requirements during the transient post-mating period is similar to "self-selection" which is a crucial and fundamental behavior for polyphagous and oligophagous animals to recognize and compensate for their insufficient nutrients from their selected diets (Richter et al., 1938). This self-selection is observed in many animals, including insects (Waldbauer and Friedman, 1991). Therefore, animals are able to select suitable nutrients to fit their dietary demands when feeding ad libitum. As observed in this study, mated female crickets preferentially selected the proteinrich diet. This behavior indicates that mated crickets shift their nutritional demands from a normal state to a specific state where they are able to select the required nutrients.

\section{CONCLUSION}

Mated female G. bimaculatus crickets tended to eat more food compared to virgin females. In addition, geometric framework analyses revealed that $G$. bimaculatus females exhibited a transient switch to a protein rich diet immediately after mating.

\section{ACKNOWLEDGMENTS}

This work was partly supported by Grant-in-Aid for Scientific Research \#24580157 from the Ministry of Education, Science, Sports, and Culture of Japan, and the NAITO Foundation.

\section{REFERENCES}

Adlakha, V., and Pillai, M. K. (1976). Role of male accessory gland substance in the regulation of blood intake by mosquitoes. J. Insect Physiol. 22, 1441-1442. doi: 10.1016/0022-1910(76)90206-7

Avila, F. W., Sirot, L. K., LaFlamme, B. A., Rubinstein, C. D., and Wolfner, M. F. (2011). Insect seminal fluid proteins: identification and function. Annu. Rev. Entomol. 56, 21-40. doi: 10.1146/annurev-ento-120709144823

Brent, C. S. (2010). Reproduction of the western tarnished plant bug, Lygus hesperus, in relation to age, gonadal activity and mating status. J. Insect Physiol. 56, 28-34. doi: 10.1016/j.jinsphys.2009.08.021

Carvalho, G. B., Kapahi, P., Anderson, D. J., and Benzer, S. (2006). Allocrine modulation of feeding behavior by the Sex Peptide of Drosophila. Curr. Biol. 16, 692-696. doi: 10.1016/j.cub.2006.02.064

Chen, P. S., Stumm-Zollinger, E., Aigaki, T., Balmer, J., Bienz, M., and Böhlen, P. (1988). A male accessory gland peptide that regulates reproductive behavior of female D. melanogaster. Cell 54, 291-298. doi: 10.1016/0092-8674(88)90192-4

Chiba, Y., Shinkawa, Y., Yoshii, M., Matsumoto, A., Tomioka, K., and Takahashi, S. Y. (1992). A comparative study on insemination dependency of circadian activity pattern in mosquitoes. Physiol. Entomol. 17, 213-218. doi: 10.1111/j.13653032.1992.tb01013.x

Cooper, W. R., and Spurgeon, D. W. (2011). Laboratory studies of variations in feeding behaviors among Lygus hesperus (Hemiptera: Miridae) of different gender and reproductive states. Environ. Entomol. 40, 367-373. doi: $10.1603 /$ EN10149

Ejezie, G. C., and Davey, K. G. (1977). Some effects of mating in female tsetse, Glossina austeni Newst. J. Exp. Zool. 200, 303-310. doi: 10.1002/jez.1402000211

Gillott, C. (2003). Male accessory gland secretions: modulators of female reproductive physiology and behavior. Annu. Rev. Entomol. 48, 163-184. doi: 10.1146/annurev.ento.48.091801.112657

Hanin, O., Azrielli, A., Zakin, V., Applebaum, S., and Rafaeli, A. (2011). Identification and differential expression of a sex-peptide receptor in Helicoverpa armigera. Insect Biochem. Mol. Biol. 41, 537-544. doi: 10.1016/j.ibmb.2011.03.004

Jang, E. B. (2002). Physiology of mating behavior in mediterranean fruit fly (Diptera: Tephritidae): chemoreception and male accessory gland fluids in female post-mating behavior. Fla. Entomol. 85, 89-93. doi: 10.1653/00154040(2002)085[0089:POMBIM]2.0.CO;2
Jin, Z. Y., and Gong, H. (2001). Male accessory gland derived factors can stimulate oogenesis and enhance oviposition in Helicoverpa armigera (Lepidoptera: Noctuidae). Arch. Insect Biochem. Physiol. 46, 175-185. doi: 10.1002/ arch. 1027

Judson, C. L. (1967). Feeding and oviposition behavior in the mosquito Aedes aegypti (L.). I. Preliminary studies of physiological control mechanisms. Biol. Bull. 133, 369-378. doi: 10.2307/1539832

Kim, Y. J., Bartalska, K., Audsley, N., Yamanaka, N., Yapici, N., Lee, J. Y., et al. (2010). MIPs are ancestral ligands for the sex peptide receptor. Proc. Natl. Acad. Sci. U.S.A. 107, 6520-6525. doi: 10.1073/pnas.0914764107

Konuma, T., Morooka, N., Nagasawa, H., and Nagata, S. (2012). Knockdown of the adipokinetic hormone receptor increases feeding frequency in the two-spotted cricket Gryllus bimaculatus. Endocrinology 153, 3111-3122. doi: 10.1210/en.2011-1533

Kubli, E., and Bopp, D. (2012). Sexual behavior: how sex peptide flips the postmating switch of female flies. Curr. Biol. 22, R520-R522. doi: 10.1016/j.cub.2012.04.058

Lawniczak, M. K., and Begun, D. J. (2004). A genome-wide analysis of courting and mating responses in Drosophila melanogaster females. Genome 47, 900-910. doi: 10.1139/g04-050

McGraw, L. A., Gibson, G., Clark, A. G., and Wolfner, M. F. (2004). Genes regulated by mating, sperm, or seminal proteins in mated female Drosophila melanogaster. Curr. Biol. 14, 1509-1514. doi: 10.1016/j.cub.2004.08.028

Nagalakshmi, V. K., Applebaum, S. W., Azrielli, A., and Rafaeli, A. (2007). Female sex pheromone suppression and the fate of sex-peptide-like peptides in mated moths of Helicoverpa armigera. Arch. Insect Biochem. Physiol. 64, 142-155. doi: 10.1002/arch.20167

Nakamura, T., Yoshizaki, M., Ogawa, S., Okamoto, H., Shinmyo, Y., Bando, T., et al. (2010). Imaging of transgenic cricket embryos reveals cell movements consistent with a syncytial patterning mechanism. Curr. Biol. 20, 1641-1647. doi: 10.1016/j.cub.2010.07.044

Peng, J., Zipperlen, P., and Kubli, E. (2005). Drosophila sex-peptide stimulates female innate immune system after mating via the Toll and Imd pathways. Curr. Biol. 15, 1690-1694. doi: 10.1016/j.cub.2005.08.048

Perry, J. C. (2011). Mating stimulates female feeding: testing the implications for the evolution of nuptial gifts. J. Evol. Biol. 24, 1727-1736. doi: 10.1111/j.14209101.2011.02299.x

Pilpel, N., Nezer, I., Applebaum, S. W., and Heifetz, Y. (2008). Mating-increases trypsin in female Drosophila hemolymph. Insect Biochem. Mol. Biol. 38, 320-330. doi: 10.1016/j.ibmb.2007.11.010

Poels, J., Van Loy, T., Vandersmissen, H. P., Van Hiel, B., Van Soest, S., Nachman, R. J., et al. (2010). Myoinhibiting peptides are the ancestral ligands of the promiscuous Drosophila sex peptide receptor. Cell. Mol. Life Sci. 67, 3511-3522. doi: 10.1007/s00018-010-0393-8

Raubenheimer, D., and Simpson, S. J. (1993). The geometry of compensatory feeding in the locust. Anim. Behav. 45, 953-964. doi: 10.1006/anbe.1993.1114

Ribeiro, C., and Dickson, B. J. (2010). Sex peptide receptor and neuronal TOR/S6K signaling modulate nutrient balancing in Drosophila. Curr. Biol. 20, 1000-1005. doi: 10.1016/j.cub.2010.03.061

Richter, C. P., Holt, L. E. Jr., Barelare, B. Jr. (1938). Nutritional requirements for normal growth and reproduction in rats studied by the self-selection method. Am. J. Physiol. 122, 734-744.

Simpson, S. J., and Raubenheimer, D. (1995). The geometric analysis of feeding and nutrition: a user's guide. J. Insect Physiol. 41, 545-553. doi: 10.1016/00221910(95)00006-G

Takami, Y., Sasabe, M., Nagata, N., and Sota T. (2008). Dual function of seminal substances for mate guarding in a ground beetle. Behav. Ecol. 19, 1173-1178. doi: 10.1093/beheco/arn090

Tatar, M. (2011). The plate half-full: status of research on the mechanisms of dietary restriction in Drosophila melanogaster. Exp. Gerontol. 46, 363-368. doi: 10.1016/j.exger.2010.12.002

Vargas, M. A., Luo, N., Yamaguchi, A., and Kapahi, P. (2010). A role for S6 kinase and serotonin in postmating dietary switch and balance of nutrients in $D$. melanogaster. Curr. Biol. 20, 1006-1011. doi: 10.1016/j.cub.2010.04.009

Waldbauer, G. P., and Friedman, S. (1991). Self-selection of optimal diets by insects. Ann. Rev. Entmol. 36, 43-63. doi: 10.1146/annurev.en.36.010191.000355

Watanabe, T., Ochiai, H., Sakuma, T., Horch, H. W., Hamaguchi, N., Nakamura, T., et al. (2012). Non-transgenic genome modifications in a hemimetabolous insect using zinc-finger and TAL effector nucleases. Nat. Commun. 3, 1017. doi: $10.1038 /$ ncomms 2020 
Wolfner, M. F. (1997). Tokens of love: functions and regulation of Drosophila male accessory gland products. Insect Biochem. Mol. Biol. 27, 179-192. doi: 10.1016/S0965-1748(96)00084-7

Wolfner, M. F., Heifetz, Y., and Applebaum, S. W. (2005). "Gonadal glands and their gene products," in Comprehensive Molecular Insect Science, eds L. Gilbert, K. Iatrou, and S. Gill (Amsterdam: Elsevier), 179-212.

Yapici, N., Kim, Y. J., Ribeiro, C., and Dickson, B. J. (2008). A receptor that mediates the post-mating switch in Drosophila reproductive behaviour. Nature 451, 33-37. doi: 10.1038/nature06483

Conflict of Interest Statement: The authors declare that the research was conducted in the absence of any commercial or financial relationships that could be construed as a potential conflict of interest.
Received: 30 October 2013; accepted: 24 February 2014; published online: 13 March 2014.

Citation: Tsukamoto Y, Kataoka H, Nagasawa H and Nagata S (2014) Mating changes the female dietary preference in the two-spotted cricket, Gryllus bimaculatus. Front. Physiol. 5:95. doi: 10.3389/fphys.2014.00095

This article was submitted to Integrative Physiology, a section of the journal Frontiers in Physiology.

Copyright (c) 2014 Tsukamoto, Kataoka, Nagasawa and Nagata. This is an openaccess article distributed under the terms of the Creative Commons Attribution License (CC BY). The use, distribution or reproduction in other forums is permitted, provided the original author(s) or licensor are credited and that the original publication in this journal is cited, in accordance with accepted academic practice. No use, distribution or reproduction is permitted which does not comply with these terms. 